\title{
Beyond behavioural intentions: Planning mediates between intentions and physical activity
}

\author{
Urte Scholz ${ }^{\prime *}$, Benjamin Schüz ${ }^{2}$, Jochen P. Ziegelmann ${ }^{3}$, \\ Sonia Lippke ${ }^{3}$ and Ralf Schwarzer ${ }^{3}$ \\ 'Department of Psychology, University of Zürich, Switzerland \\ ${ }^{2}$ German Centre of Gerontology, Berlin, Germany \\ ${ }^{3}$ Freie Universität Berlin, Germany
}

\begin{abstract}
Objectives. Health-behaviour change is not predicted well by behavioural intentions alone. Action planning and coping planning are two self-regulatory strategies that may help bridge the gap between intentions and behaviour. Action planning comprises the when, where and how of implementing a behaviour. Coping planning involves the anticipation of barriers and ways to overcome them. The study examines whether the inclusion of these two planning constructs would improve the overall prediction of physical activity. The main research question is which kind of planning would be beneficial for the initiation of behaviour and which one for its maintenance. Moreover, it is examined whether intentions moderate the planning-behaviour relationship.

Methods. A 5-week longitudinal on-line study was conducted with 354 participants. Differences in prediction patterns between formerly active and formerly inactive individuals were compared using multigroup structural equation modelling. Moderating effects of intentions were tested by means of latent interactions.

Results. Inclusion of the two planning factors accounted for substantially more behaviour variance than intention alone did. Coping planning mediated the intentionbehaviour relationship only in formerly active, but not in formerly inactive participants. Moreover, intentions moderated the effects of both types of planning on behaviour.

Conclusions. Action planning appears to predict behaviour only when intentions are high. For actors, coping planning represents a critical self-regulation strategy to maintain their physical activity levels.
\end{abstract}

In order to reduce cardiovascular risk and to promote overall health, regular vigorous physical activity is strongly recommended (Lee \& Paffenbarger, 1998; Sesso, Paffenbarger, \& Lee, 2000; U.S. Department of Health and Human Services, 1996). However, most individuals engage in much less physical activity than recommended (Schoenborn, Adams, Barnes, Vickerie, \& Schiller, 2004). Regular physical activity is a

* Correspondence should be addressed to Urte Scholz, University of Zurich, Department of Psychology, Social- and Health Psychology, Binzmuehlestr, 14/Box 14, CH-8050 Zürich, Switzerland (email: urte.scholz@psychologie.uzh.ch). 
complex behaviour consisting of many behavioural facets and complex sequences that need to be maintained on a long term, at best lifelong basis. Thus, changing from inactivity to regular activity constitutes a complex process with different tasks during change. These tasks differ from those involved in changing less complex health behaviours, such as making a doctor's appointment. Moreover, the majority of individuals fail to actually change a sedentary life-style into an active one, although their motivation is high. This holds true even for high-risk groups, such as cardiac patients, for whom behaviour change is mandatory in order to prevent further harm (Dusseldorp, Van Elderen, Maes, Meulman, \& Kraaij, 1999).

\section{Predicting health-behaviour change}

Intentions not only comprise a person's motivation towards a goal in terms of direction and intensity, but also a necessary prerequisite for life-style changes (Sheeran, 2002). According to most prevailing theories of health-behaviour change, such as the Theory of Planned Behaviour (TPB; Ajzen, 1991) and Protection Motivation Theory (PMT; Maddux \& Rogers, 1983), intentions are the best predictors of behaviour. However, metaanalyses have shown that intentions alone are not sufficient to predict behavioural change satisfactorily (e.g. Sheeran, 2002), as they leave large amounts of behavioural variance unexplained. This phenomenon has been labelled 'intention-behaviour gap'.

Research suggests that the prediction of behaviour change can be improved by furnishing intentions with self-regulatory skills that help translate these intentions into behaviour. These self-regulatory skills include perceived self-efficacy (Bandura, 2004) and planning (Leventhal, Singer, \& Jones, 1965; synonymous to implementation intentions; Gollwitzer, 1999).

Empirically, the intention-behaviour gap seems to emerge mainly in individuals who have intentions, but who subsequently fail to act on them (i.e. 'inclined abstainers', Orbell \& Sheeran, 1998). This indicates that forming intentions and intention implementation are different processes. The Health Action Process Approach (HAPA; Schwarzer, 1992; Schwarzer et al., 2007) provides a theoretical framework for this idea of distinct processes by distinguishing different stages, each stage with distinct factors relevant for single steps towards health-behaviour change (Lippke, Ziegelmann, \& Schwarzer, 2004b, 2005). Stage models construe the process of health-behaviour change as a transition through a sequence of qualitative different and distinct stages (Weinstein, Rothman, \& Sutton, 1998). It is assumed that individuals who are in the same stage experience similar barriers, and those in different stages experience different barriers to stage transition. As a consequence, this implies that there are factors that are ineffective in one stage or phase, but that are of crucial importance in another phase (Weinstein et al., 1998).

Within all stages, different patterns of social-cognitive predictors may emerge. In the motivation phase, a person develops an intention to act. Here, core assumptions of socialcognitive theory (e.g. Bandura, 2004) are incurred. The HAPA assumes risk awareness to be a distal antecedent of intention formation within the motivation phase. It sets the stage for a contemplation process and further elaboration of thoughts about consequences of behaviour and subjective competences. During this contemplation process, outcome expectancies (e.g. 'If I exercise regularly, I will lose weight') and self-efficacy regarding this behaviour (e.g. 'I will be able to adhere to my exercise schedule in spite of the temptation to stay at home') facilitate intention formation. Several studies provide evidence for this assumption with regard to different samples and behaviours (e.g. Lippke, Ziegelmann, \& Schwarzer, 2004a, 2004b; Sniehotta, Scholz, \& Schwarzer, 2005; Schwarzer et al., 2007). 
After an intention has been formed, individuals enter the volitional stage, in which the intention has to be translated into action. Subsequent to forming an intention, different tasks need to be mastered, such as initiating the target behaviour, maintaining it and recovering from lapses. Self-regulation skills are crucial for the uptake and maintenance of behaviour changes. Planning is one such self-regulatory skill. It is generally understood as the mental simulation of behaviour in order to be prepared for situations in which the behaviour should be performed (Lachman \& Burack, 1993). It is an economic (i.e. time and energy saving) and effective way of fostering goal attainment (Gollwitzer \& Sheeran, 2006). Studies on the translation of intention into behaviour via planning suggest that planning mediates between intention and behaviour only if individuals intend to perform certain behaviour (Lippke et al., 2005). In the present study, we differentiate between two kinds of planning: action planning (Gollwitzer, 1999; Leventhal et al, 1965) and coping planning (Sniehotta, Schwarzer, Scholz, \& Schüz, 2005; Ziegelmann, Lippke, \& Schwarzer, 2006).

\section{Action planning}

Action planning specifies the when, where and how of implementing an intended behaviour (Gollwitzer, 1999; Leventhal et al., 1965). An example for a concrete action plan might be 'Tuesdays at seven o'clock I will go running in the park for half an hour'. Action planning links behavioural responses to situational cues and thus facilitates initiation of behaviour (for an overview, see Sheeran, Milne, Webb, \& Gollwitzer, 2005). This is due to automatic elicitation of behavioural responses without conscious intent triggered by the situational cues of the action plans. Action planning is understood as a process that comes into play after an intention has been formed. Comparing the behavioural effects of intentions alone to intentions plus action planning, it has been repeatedly shown that action planning leads to a higher likelihood that the behaviour will be implemented, rather than intentions alone (Gollwitzer \& Sheeran, 2006). The usefulness of action planning for health-behaviour change has been demonstrated in different domains, such as tetanus vaccination (Leventhal et al., 1965), breast selfexamination (Luszczynska \& Schwarzer, 2003; Prestwich et al., 2005), physical activity (Hallam \& Petosa, 2004; Milne, Orbell, \& Sheeran, 2002) and nutrition (Verplanken \& Faes, 1999). However, as Jackson and colleagues (Jackson et al., 2005) demonstrated, it is unclear so far whether the effectiveness of action planning is related to the complexity of health behaviours (simple behaviours are, e.g. attending a medical screening appointment and complex behaviours are, e.g. regular physical activity). Moreover, most of the studies on planning examine a rather brief time-span. As Jackson and colleagues demonstrated, action planning was not effective in changing fruit and vegetable consumption (a complex behaviour) over a time-span of 3 months (i.e. a rather long time-span in comparison to most studies of health-behaviour change). Other studies also failed to find effects of action planning on physical activity over longer time-spans (e.g. Sniehotta, Scholz, Schwarzer, Fuhrmann et al., 2005). This might be directly related to the complexity of the target behaviour.

\section{Coping planning}

Coping planning refers to anticipating difficulties or barriers that might hinder the implementation of one's behavioural intentions. It includes the detailed planning on how to overcome such difficulties (Sniehotta, Schwarzer et al., 2005; Ziegelmann 
et al., 2006). An example for a coping plan could be: 'If it rains heavily, I will go to the gym instead of going running'. The mnemonic and attentual pathways operating here are the same as in action planning: an if-condition is linked to a then-component (behavioural response). Thus, by making coping plans, individuals anticipate how they control unwanted distractions (if-condition) and how they cope with difficulties that interfere with the execution (then-component) (Patterson \& Mischel, 1976). Similar approaches are used in cessation programs for addictive behaviours (Marlatt, 1996). In fact, two core ingredients of Marlatt's relapse prevention model are (a) the identification of high-risk situations in which it is most likely for patients to relapse into their old habits (if-condition) and (b) the generation of appropriate behavioural responses to cope with these situations (then-component; Larimer, Palmer, \& Marlatt, 1999; Marlatt, 1996). Coping planning, however, is less comprehensive and elaborated than the entire coping skills training suggested by Marlatt. It comprises the anticipation of high-risk situations and the mental simulation of coping with these situations. Coping planning has been found useful in changing complex health behaviours, such as regular physical exercise (e.g. Simkin \& Gross, 1994; Sniehotta, Scholz, Schwarzer, Fuhrmann et al., 2005; Ziegelmann et al., 2006).

\section{Stage-specific effects of planning}

Action planning and coping planning are conceptualized as being two distinct theoretical constructs. Whereas action planning is more useful for the initiation of complex behaviours, coping planning is assumed to facilitate behaviour maintenance. Studies on physical exercise in cardiac and orthopaedic rehabilitation patients provide evidence for these assumptions: In a study with cardiac rehabilitation patients, coping planning yielded a significant effect on change in physical activity only from 2 to 4 months after discharge from rehabilitation, that is, after participants had already initiated a physical exercise regimen and were then challenged with maintaining it (Sniehotta, Schwarzer et al., 2005). In a study with orthopaedic rehabilitation patients, Ziegelmann et al. (2006) demonstrated that action planning predicted behaviour from the beginning of the behaviour change process, whereas coping planning resulted in a delayed effect by predicting change in physical activity at the 4 -week and the 6 -month follow-up, but not at an initial 2-week follow-up. These studies provide evidence that coping planning is an important strategy for maintaining complex behaviour, rather than for its initiation. Previous phase-specific studies (e.g. Lippke et al., 2004b, 2005) did not differentiate between action planning and coping planning. And previous research on action planning, and coping planning has not explicitly examined distinct effects of both planning concepts during different phases of the HAPA model (e.g. Sniehotta, Schwarzer et al., 2005; Ziegelmann et al., 2006). Both aspects were included for the first time in the current study.

Moreover, in accordance with the assumptions of stage models, recent studies have demonstrated that action planning is most effective in postintentional individuals (Lippke et al., 2004b; Sheeran, Webb, \& Gollwitzer, 2005). For example, a study by Sheeran and colleagues (Sheeran, Webb et al., 2005) demonstrated that implementation intentions were only beneficial for subsequent behaviour when the respective goal intentions were activated. Likewise, in a two-study paper by Norman and Conner (2005), both studies provided evidence for an interaction between intentions and planning on exercise behaviour. In Study 1, the planning-behaviour relation was only significant when intentions were high, but not when intentions 
were low or moderate. In Study 2, the same pattern emerged in that planning was only significantly related to behaviour when intentions were moderate to strong, but not when they were low.

It is assumed that this interaction with intentions also applies for coping planning in that both types of planning should be most effective for behaviour change when a respective goal intention has been formed (i.e. when people are in the postintentional volitional stage of behaviour change). Thus, we aimed at testing whether the hypothesized differential effects of action planning and coping planning in terms of initiation and maintenance of behaviour might be pronounced when intentions are strong.

\section{Aims of this study}

The aim of our study was threefold. First, we aimed at testing whether action planning and coping planning improved the prediction of change in physical activity over and above the effect of behavioural intentions. Second, we tested the hypothesized differential effects of action planning and coping planning on initiation and maintenance of physical activity. Action planning is assumed to prompt the initiation of behavioural change, whereas coping planning should facilitate its maintenance. And third, we were interested in whether intentions moderated the planning-behaviour relationship in the way that action planning and coping planning may display stronger associations with behaviour when intentions are strong. Further, we were interested whether this again was different for initiators and maintainers.

\section{Method}

\section{Sample and procedure}

An on-line study was launched using the software dynQuest (Rademacher \& Lippke, 2007). The link to the html-pages of the on-line study was placed on several websites of life-style magazines as well as on a website of the Health Psychology Department of the Freie Universität Berlin, Germany. At the first point of measurement, 991 participants completed the on-line questionnaires. The second point of measurement was implemented 5 weeks later (Time 2) by inviting participants by e-mail to fill out the follow-up questionnaire. The Time 2 questionnaire was completed by 354 participants. The high drop-out rate (64\%) is quite common in on-line studies due to anonymity and the difficulty to follow-up participants (e.g. misspelled or missing e-mail addresses; Kraut et al., 2004; Reips, 2002). Drop-out analyses revealed that the longitudinal sample did not differ from the original sample on the study variables, but drop-outs were on average about 2 years younger than participants of the longitudinal sample, $F(1,919)=8.33, p<.05$.

Participants of the longitudinal sample were mostly women (81.4\%) and had a mean age of 37 years, $S D=9.90$, range 16-64 years. The majority of the participants were married, $N=203,57.3 \%, 31.9 \%$ were single, $7.9 \%$ were divorced and 7 persons $(2.0 \%)$ were widowed. Three participants $(0.8 \%)$ did not indicate their marital status. The majority of participants had attended 13 years of school, $N=255,72.0 \%, 21(6.0 \%)$ participants had attended 12 years, $65(18.4 \%) 9$ years, $4(1.1)$ had 8 years, $3(0.8 \%)$ participants had not yet finished school and $6(1.7 \%)$ participants did not report about their education. 


\section{Measures}

Social-cognitive variables were assessed at Time 1 , together with past vigorous physical exercise. If not indicated otherwise, scales are taken from Schwarzer et al. (2007). The response format was a four-point Likert scale ranging from 1 (disagree) to 4 (agree). All means and standard deviations are displayed in Table 1.

Table I. Intercorrelations between latent variables included in the study

\begin{tabular}{|c|c|c|c|c|c|c|c|c|c|c|}
\hline & & 1 & 2 & 3 & 4 & 5 & 6 & 7 & Mean & SD \\
\hline 1 & Risk awareness & & & & & & & & 2.44 & 0.86 \\
\hline 2 & Positive outcome expectancies & $.18 *$ & & & & & & & 3.60 & 0.44 \\
\hline 3 & Self-efficacy & $-.28 * *$ & $.35 * *$ & & & & & & 3.30 & 0.57 \\
\hline 4 & Intentions & -.08 & $.38 * *$ & $.70 * *$ & & & & & 3.40 & 0.66 \\
\hline 5 & Action planning & $-.17 *$ & $.17^{*}$ & $.39 \% *$ & $.50 * *$ & & & & 3.30 & 0.71 \\
\hline 6 & Coping planning & $-.22 * *$ & .09 & $.40 * *$ & $.32 * *$ & $.57 * *$ & & & 2.18 & 0.76 \\
\hline 7 & $\begin{array}{l}\text { Time I vigorous physical } \\
\text { activity }\end{array}$ & $-.37 * *$ & -.01 & $.40^{* *}$ & $.35 * *$ & $.27^{* *}$ & $.36 * *$ & & 73.00 & 81.05 \\
\hline 8 & $\begin{array}{l}\text { Time } 2 \text { vigorous physical } \\
\text { activity }\end{array}$ & $-.22 * *$ & .03 & $.43 * *$ & $.37 * *$ & $.28 * *$ & $.39 * *$ & $.69 * *$ & 83.86 & 91.39 \\
\hline
\end{tabular}

Note. Numbers 1-7 measured at Time 1; Time $2=5$-week follow-up.

$* p<.05 ; * p<.01$.

Risk awareness was measured at Time 1 by three items (Cronbach's $\alpha=.80$ ) assessing situation-outcome expectations, for example: 'If I keep my life-style the way it is, there is a high likelihood that I will develop severe health problems'.

Outcome expectancies regarding behaviour change were assessed at Time 1 with three items (Cronbach's $\alpha=.63$ ). All items had the stem 'If $I$ will exercise on a regular basis, . . . followed by positive consequences, such as ' . . then it will be good for my health'.

Self-efficacy was assessed by three items (Cronbach's $\alpha=.67$ ), for example, 'I am confident that I can adjust to a physically active life-style' or 'I am confident that I can be physically active at least three times a week for 30 minutes each time'.

Bebavioural intentions were measured by three items (Cronbach's $\alpha=.76$ ). The stem 'I intend to ...' was followed by the recommended activities, for example, '. . . engage in vigorous physical activities (e.g. jogging, swimming, etc.) on a regular basis'.

Action planning was assessed by four items (Cronbach's $\alpha=.85$ ). The item stem 'I have made a detailed plan regarding. . ' was followed by the items (a) '. . . when to exercise', (b) '. . . where to exercise', (c) '. . . how to exercise' and (d) '. . . how often to exercise'.

Coping planning was assessed by four items (Cronbach's $\alpha=.87$ ) using a scale from Sniehotta, Schwarzer et al. (2005). For coping planning, the same stem as for action planning was used: 'I have made a detailed plan regarding. . .', which was followed by the items '. . . what to do when something interferes with my plans', '. . how to cope with setbacks', '. . . how to stick to my intentions, even in difficult situations' and ' . . when to be especially careful in order to avoid setbacks'.

Vigorous physical exercise at Time 1 and Time 2 was assessed by using one item of the International Physical Activity Questionnaire (IPAQ; Booth, 2000). Participants were 
asked to indicate how often during the past 7 days they had engaged in vigorous physical activities such as jogging, swimming and cycling. Moreover, they were asked how much time they had usually spent performing these activities on one of those days. Frequency and average duration per session were then multiplied in order to obtain a measure of weighted duration of vigorous physical exercise during the last 7 days.

\section{Data Analysis}

Structural Equation Modelling with AMOS 6.0 using Maximum Likelihood (ML) estimation was used to test the structural assumptions. Missing values were treated using Full Information Maximum Likelihood (FIML; Arbuckle, 1996). Model fit was assessed by examining chi-squared, the comparative fit index (CFI) and the root mean square error of approximation (RMSEA). A satisfactory model fit is indicated by high CFI $(>.90)$, low RMSEA $(<.08)$ (Kline, 2005). The chi-squared of the model, however, is a sample-size-dependent index. Therefore, it cannot be considered as a basic criterion for the acceptance or rejection of a model (Hu \& Bentler, 1998).

To test whether action planning and coping planning could contribute to the prediction of Time 2 physical activity over and above behavioural intentions, three recursive models were tested. All variables except vigorous physical exercise were specified as latent variables using the above-mentioned items as manifest indicators. The first model (see Figure 1) specified the motivational constructs of the HAPA model with risk awareness, outcome expectancies, and self-efficacy being intercorrelated and specified as latent predictors for behavioural intentions. The latter, in-turn, was specified as the only predictor of Time 2 physical activity. In the second model (see Figure 2 ), the volitional constructs action planning and coping planning were added to the model to improve prediction of Time 2 vigorous physical exercise. Direct effects of intentions on action planning and coping planning were added. Action planning and coping planning were allowed to correlate. In a third model, we tested whether intentions, action planning and coping planning also predicted change in behaviour by testing Model 2 with baseline behaviour included in addition.

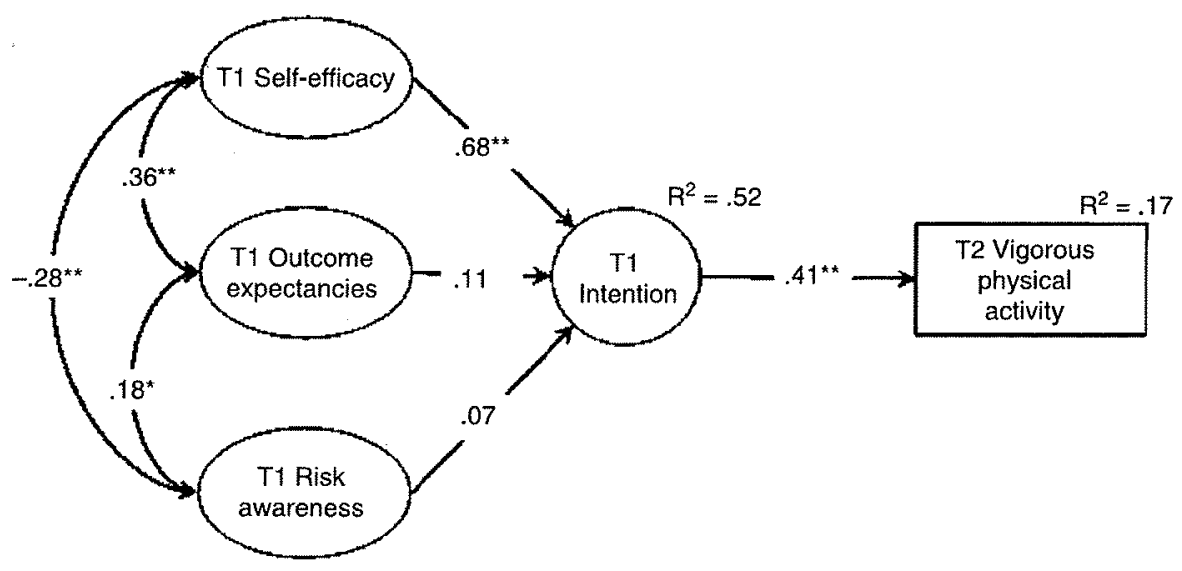

Figure I. Predicting Time 2 vigorous physical activity by intentions.

Note. $* p<.05 ; * p<.001 ; \mathrm{TI}=$ Time I baseline; T2 = Time 2, 5 weeks after Time I. 


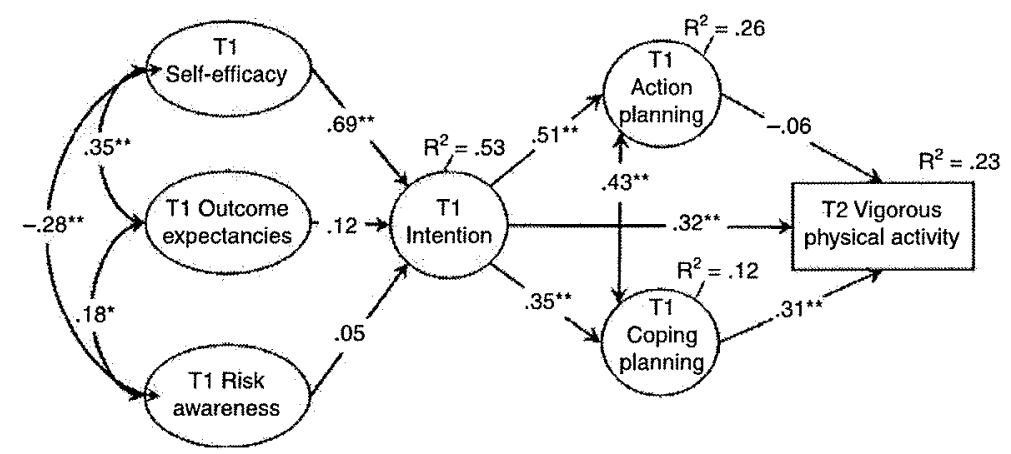

Figure 2. Predicting Time 2 vigorous physical activity by intentions, action planning and coping planning.

Note. $* p<.05 ; * p<.001 ; \mathrm{TI}=$ Time I baseline; $\mathrm{T} 2=$ Time 2,5 weeks after Time I.

A fourth model tested whether action planning is more important for action initiation, whereas coping planning is more important for maintenance. Therefore, a two-group nested model comparison was specified. The two groups were defined as follows: an inactive group that comprised $82(30 \%)$ participants who reported not having been engaged in any vigorous physical exercise in the past, as opposed to an active group of 195 participants (70\%) who had been involved in past vigorous physical exercise. We tested whether the effects of intentions, action planning and coping planning on Time 2 vigorous physical exercise differed between these two groups. This was done by fixing the respective paths for both groups and comparing them to the model with freely estimated paths. A significant chi-squared difference test indicates that the nested model (i.e. the one with more fixed effects) is not appropriate, whereas a non-significant chi-squared difference test indicates that this model fits the data as well as the model without the assumption of equal effects between-groups.

To test whether intentions would moderate the planning-behaviour relations, we followed the latent interaction approach of an unconstrained model suggested by Marsh and colleagues (Marsh, Wen, \& Hau, 2004). These authors suggest testing for latent interactions by multiplying mean-centred indicators of predictor and moderator and specifying these products as indicators of the latent interaction factor. As we had three intention items, but four items for each of the planning constructs, we generated three indicators of the latent interaction by multiplying items with similar factor loadings on latent predictor and moderator variables with each other (Marsh et al., 2004).

As we were only interested in differential effects of the volitional phase, we excluded all three motivational variables from the interaction models. We then tested two separate models. The first one included intentions, action planning, coping planning and physical activity at Time 2 and the latent interaction between action planning and intentions. The second one included all of these factors, but as interaction the latent interaction between coping planning and intentions. The two interaction effects were tested separately as we were not interested in competing effects of these two interactions.

Finally, we probed whether the assumed moderating effect of intentions was different for people who had to initiate the behaviour (i.e. former inactive group) and maintainers (i.e. former active group). Thus, we tested the two interaction models 
(after excluding baseline physical activity to avoid problems with limited variance) for initiators and maintainers using the same group comparison method as described above.

\section{Results}

Correlations between all variables in the model are displayed in Table 1. Almost all variables of the HAPA were significantly associated with each other. Moreover, all variables except positive outcome expectancies were significantly related to past vigorous physical activity and to Time 2 vigorous physical activity.

Vigorous physical activity displayed a high stability over the 5-week period, indicated by a bivariate association of $r=, 69$. There was no significant increase in vigorous physical activity over time for the total sample, $F(1,235)=2.26, p=.13, \eta^{2}=.01$.

\section{Predicting time 2 vigorous physical activity from intentions}

The first model tested whether the motivational HAPA variables predicted behavioural intentions and, additionally, to obtain the direct effect of intentions on Time 2 physical activity. Specifying intentions as predictor of physical activity reflects the core assumption of other models of health behaviours, such as the TPB (Ajzen, 1991) or PMT (Maddux \& Rogers, 1983). Figure 1 displays the first model.

Risk awareness and positive outcome expectancies were not significantly related to behavioural intentions. Self-efficacy, in contrast, was closely related to behavioural intentions, $\beta=0.68, p<.001$. All in all, $52 \%$ of variance in behavioural intentions was accounted for. Intentions, in-turn, accounted for $17 \%$ of variance in vigorous physical activity 5 weeks later. The model fit was satisfactory, with CFI $=.94$, RMSEA $=.06(90 \%$ $\mathrm{CI}=.048, .075), \chi^{2}=138.32 ; d f=59, p<.001$

Predicting time 2 vigorous physical activity jointly by intentions, action planning and coping planning

In the second model, Time 1 action planning and coping planning were added to the model to find out whether the inclusion of these two volitional constructs improved the prediction of behaviour over and above the effect of intentions. Behavioural intentions predicted both action planning and coping planning significantly (see Figure 2). However, action planning did not have a significant effect on Time 2 physical activity. Coping planning, in contrast, was a strong significant predictor of Time 2 vigorous physical activity. The indirect effect of intentions on Time 2 physical activity via coping planning was $\beta=0.11$ and was statistically significant as indicated by the Sobel test (Sobel, 1982), $Z_{\text {sobel }}: 3.23, p<.001$. The direct effect of intentions on Time 2 physical activity remained significant. The amount of explained variance in Time 2 vigorous physical activity was enhanced to $23 \%$. Model fit again was satisfactory, with CFI $=.95$, RMSEA $=.05(90 \% \mathrm{CI}=.041, .058), \chi^{2}=333.47 ; d f=178, p<.001$.

\section{Predicting change in vigorous physical activity}

In a third model, Time 1 physical activity was included in order to test for the prediction of change in physical activity from Time 1 to Time 2 . All motivational and volitional variables were allowed to correlate with past behaviour. As past behaviour had a strong effect on physical activity at Time $2, \beta=0.60, p<.001$, the effects of intentions and 
coping planning on Time 2 behaviour found in the second model decreased in size, but remained significant. Behavioural intentions displayed an association with change in behaviour of $\beta=0.16, p=.01$ and coping planning exhibited an effect on change in behaviour of $\beta=0.13, p=.02$. Likewise, the indirect effect from intentions over coping planning on change in behaviour was only $\beta=0.04$, but still reached significance, $Z_{\text {sobel }}: 2.07, p=.039$. Action planning was not significantly associated with change in physical activity, $\beta=-0.02, p=.74$. The model fit was satisfactory, with $\mathrm{CFI}=.95, \mathrm{RMSEA}=.05(90 \% \mathrm{CI}=.039, .055), \chi^{2}=345.19 ; d f=192, p<.001$.

Differences in prediction patterns between former inactive and active individuals The fourth model tested our assumptions that action planning was more important for the initiation of behaviour, whereas coping planning was more important for maintenance. Therefore, we compared the effects of intentions, action planning and coping planning for inactive participants (initiators) and active participants (maintainers) by fixing the effect parameters of intentions, action planning and coping planning on behaviour to be equal across groups.

The two-group nested model comparison resulted in a non-significant chi-squared difference test for the assumption that the effects of intentions and of action planning on behaviour were equal across groups when compared with the freely estimated default model, $\chi^{2}$ difference $=4.05, d f=1, p=.13$. Thus, effects of intentions and action planning on Time 2 physical activity were not statistically different for initiators, $\beta$ for intentions on Time 2 physical activity $=.22, p=.08 ; \beta$ for action planning on Time 2 physical activity $=-.004, p=.98$, and maintainers, $\beta$ for intentions on Time 2 physical activity $=.11, p=.08 ; \beta$ for action planning on Time 2 physical activity $=-.002$, $p=: 98$. This indicates that our assumption does not hold that action planning might be of particular value to initiate the behaviour in the former inactive group.

Adding a fixed effect for coping planning that is assuming the effect of coping planning to be equal across groups resulted in a highly significant chi-squared difference test when compared with the model with intentions and action planning being equal across groups, $\chi^{2}$ difference $=14.35, d f=1, p=.003$. Thus, the effect of coping planning is different for initiators and maintainers. For initiators, coping planning did not have a significant effect on Time 2 physical activity, $\beta=-0.07, p=.63$, but for maintainers the effect was strong and significant, with $\beta=0.36, p<.001$. For maintainers, coping planning was confirmed to act as a mediator between intentions and Time 2 activity, with the indirect effect of $\beta=0.07$, using the Sobel test, $\mathrm{Z}_{\text {sobel }}=2.03, p=.048$. Thus, the hypothesis was confirmed that, for formerly active people who were about to maintain the behaviour, coping planning is more important than for individuals who need to initiate a behaviour change.

The two groups differed significantly in their average Time 2 physical activity, $F(1,234)=53.76, p<.001$, Cohen's $d=1.21$, indicating a strong effect. The former inactive group reported a mean of $22.22, S D=46.14$, the maintainers a mean of 110.35 , $S D=91.86$. The mean difference between the two groups was $-88.13,95 \%$ $\mathrm{CI}=-111.81,-64.45$. Likewise, significant mean differences between the two groups emerged for Time 1 action planning, $F(1,275)=17.65, p<.001$, Cohen's $d=.52$, mean difference $=-.38,95 \% \mathrm{CI}=-.56,-.20$, and Time 1 coping planning, $F(1,275)=24.87, \quad p<.01$, Cohen's $d=.68$, mean difference $=-.48, \quad 95 \%$ $\mathrm{CI}=-.66,-.31$. Maintainers consistently reported higher mean levels, action planning: $M=3.42, S D=0.65$; coping planning: $M=2.34, S D=0.77$, than the 
initiators, action planning: $M=3.05, S D=0.77$; coping planning: $M=1.86, S D=0.63$, albeit with the difference in action planning being on a high mean level for both groups.

\section{Testing for differential effects of planning depending on intention strength}

Finally, we tested whether intentions moderated the planning-behaviour relation. As intentions were measured continuously, we refrained from a multigroup comparison based on arbitrary cut-off values, but we specified a latent interaction model (Marsh et al., 2004).

First, we probed the interaction between action planning and intentions on behaviour. Action planning, intentions (with mean-centered indicators), the latent interaction thereof and coping planning were specified as predictors of Time 2 physical activity. All predictors of behaviour at Time 2 were allowed to correlate. The model showed a non-significant effect of action planning on behaviour, $\beta=0.06, p=.54$, a significant main effect of intentions, $\beta=0.38, p<.001$, a significant effect of coping planning, $\beta=0.28, p<.001$, and a significant effect of the latent interaction between action planning and intentions, $\beta=0.25, p<.001$. The interaction confirmed our assumptions: The association between action planning and behaviour increased with increasing levels of intentions. Model fit was satisfactory with $\mathrm{CFI}=.96$, RMSEA $=.06$ $(90 \% \mathrm{CI}=.048, .071), \chi^{2}=182.84 ; d f=81, p<.001$.

In a second step we explored this interaction effect for initiators and maintainers. We tested whether the effect of the interaction on behaviour at Time 2 could be fixed to be equal for both groups in a nested model comparison. The unstandardized effect could be assumed equal for both groups, $\chi^{2}$ difference $=2.39, d f=1, p=.12$, with $\beta=0.34, p=.09$, for initiators and $\beta=0.09, p=.09$, for maintainers. The interaction effects within the two groups qualified for the whole sample with stronger associations between action planning and behaviour when higher intentions were reported. In terms of main effects, the same picture emerged as in the former two-group model, with action planning not significantly predicting behaviour for both groups, and coping planning and intentions only significantly predicting behaviour in the maintaining group.

We then tested the latent interaction between coping planning and intentions on Time 2 physical activity, including the same volitional predictors as above. A significant effect of coping planning also emerged here, $\beta=0.26, p<.01$, as well as of intentions, $\beta=0.33, p<.001$, but not of action planning, $\beta=0.02, p=.79$. The latent interaction was also significant, $\beta=0.18, p<.05$. Similar to action planning, the coping planningbehaviour relationship increased with stronger intentions. This again is in line with our hypothesis. Model fit was satisfactory, with $\mathrm{CFI}=.94, \quad \mathrm{RMSEA}=.07 \quad(90 \%$ $\mathrm{CI}=.057, .080), \chi^{2}=214.97 ; d f=81, p<.001$. These effects, however, did not hold when testing for group differences between formerly inactive and active individuals. Here, the interaction effect could be fixed to equal values for both groups, $\chi^{2}$ difference $=.00, d f=1, p=.99$, and was no longer significant, $\beta=0.03, p=.74$ for initiators and $\beta=0.02, p=.74$ for maintainers. Again, main effects of coping planning, $\beta=0.32, p=.003$, and intentions, $\beta=0.20, p=.024$, emerged only for the maintainers; no significant effects emerged for the former inactive (i.e. initiators).

\section{Discussion}

Our study confirmed the assumptions of the HAPA model for the prediction of health behaviour, which is in line with previous studies (e.g. Lippke et al., 2004a, 2005; Sniehotta, Scholz, Schwarzer, Fuhrmann et al., 2005; Schwarzer et al., 2007; Ziegelmann 
et al., 2006). Risk awareness, positive outcome expectancies and self-efficacy accounted for $52 \%$ of variance in behavioural intentions. This is comparable with the prediction of intentions when applying the constructs of the TPB (e.g. Godin \& Kok, 1996). Moreover, the inclusion of action planning and coping planning resulted in an improved prediction of behaviour. This again is in line with previous studies (e.g. Schwarzer et al, 2007).

The results of our study contribute to research on health-behaviour change by demonstrating the importance of coping planning. By comparing formerly inactive participants with previously active individuals, the findings suggest different stages in the health-behaviour change process and identify key constructs within these different stages. This confirms differentiating between a motivational and a volitional phase and subdividing the latter into initiation and maintenance as in the HAPA (Sutton, 2005; Weinstein et al., 1998). Although the interaction effect investigated here is not a strict test of a stage model (Weinstein et al., 1998), our study nevertheless supports the idea of qualitatively different stages in the process of health-behaviour change by examining differential effects of a factor (i.e. coping planning) in two stage groups as defined by previous behaviour. More specifically, results suggest that coping planning is especially important for behaviour maintenance because it mediated the effects of intentions only in the subsample of previously active individuals who were about to maintain the behaviour. Moreover, the mean difference indicates that it is being used to a higher extent by maintainers than by individuals who need to initiate the behaviour first. This is in line with previous research (Sniehotta, Schwarzer et al., 2005; Ziegelmann et al., 2006).

The assumption of different stages was also supported by the test of the interaction of intentions and action planning and coping planning, respectively. This was guided by the idea that planning is more predictive for behaviour in postintentional individuals (i.e. when paired with high intentions; Sheeran, Webb et al., 2005). Besides the strong and consistent main effect of coping planning on behaviour, coping planning was even more strongly associated with behaviour when intentions were high. This pattern however did not emerge when formerly inactive individuals and formerly active individuals were compared. Here, only the maintainers profited from coping planning irrespective of intention strength, whereas it was not at all important for formerly inactive individuals who were about to initiate the behaviour. Those persons in our study who were formerly inactive might rather have had to cope with initiating behaviour than with overcoming difficulties in maintaining the exercise regimen. However, these findings need replication with other samples before final conclusions can be drawn.

Coping planning comprises the anticipation of difficulties or barriers, that is, risk situations that might hinder an individual to act upon his or her intentions, together with a concrete plan on how to overcome the critical situations. Coping plans are part of the more comprehensive coping skill training (Marlatt \& Gordon, 1985) introduced for maintaining behaviour change in addiction therapy. This places coping planning in line with the literature on relapse prevention (see Marlatt, 1996).

The non-significant main effect of action planning on Time 2 physical activity for the maintaining group was in line with our hypotheses because we did not expect those who were already active to be in need of strategies for initiating a new behaviour. However, there was also no main effect of action planning on physical activity for the initiating group. Examining the moderating role of intentions, another picture emerged. Both groups, nonactors as well as actors, benefited from action planning only if intentions were high. This is in line with previous research on the moderating role of 
intentions on the effects of action plans on behaviour (Sheeran, Webb et al, 2005; Lippke et al, 2004b). Moreover, the standardized effect was slightly stronger for the formerly inactive participants than for the formerly active ones. Although these results have to be interpreted with appropriate caution, they suggest a trend in the presumed direction that inactive individuals benefit more from action planning than actors (i.e. the active group).

Some limitations in our study need to be addressed: there was a high drop-out rate, which, however, was unrelated to the core constructs of the study. A second limitation was the assessment of physical activity by self-report, although there is evidence that self-reports of physical activity are valid (Miller, Freedson, \& Kline, 1994). Moreover, the distribution of the follow-up measure of vigorous physical activity was skewed. This is in line with the majority of measures and studies on this topic (Sallis \& Saelens, 2000). Yet, the normal probability plot of the standardized residuals indicated that assumption of normality of residuals was reasonable for this measure in the overall group as well as for both subgroups.

This study has several implications for future research. There are already some studies investigating the differential effects of action planning and coping planning on complex behaviour change (e.g. Ziegelmann et al., 2006). Additional ones examining the effects of coping planning on longer-term maintenance are needed. For example, applying a longitudinal design over a time-span of at least 1 year might benefit from considering possible moderators as indicators for different phases of the healthbehaviour change process. Similar studies have been implemented successfully with differential effects of different kinds of phase-specific self-efficacy (e.g. Scholz, Sniehotta, \& Schwarzer, 2005).

Action planning and coping planning can easily be implemented in interventions to foster behaviour change in complex health behaviours (e.g. physical exercise; Ziegelmann et al., 2006). Research also suggests that a combined action planning plus coping planning intervention for physical activity adherence has differential effects in intenders (participants intending to change behaviour) and actors (participants already active) (Lippke et al., 2004b). However, this was the first study so far that examined differential effects of action planning and coping planning in terms of stages. Thus, there is a need for studies that explicitly test the differential effects of action planning and coping planning interventions for persons in different stages of the health-behaviour change process. Likewise, similar studies should target other health behaviours to find out whether more complex behaviours, such as smoking or nutrition, need different strategies in order to facilitate changes than less complex behaviours, such as adherence to annual cancer screening or dental flossing.

To conclude, our study provides evidence for the usefulness of including volitional components into the prediction of physical activity over- and above-behavioural intentions. Moreover, based on the assumption of different stages of behaviour change, these volitional components might unfold their effects differently, depending on which respective phase the individuals are in.

\section{References}

Ajzen, I. (1991). The theory of planned behavior. Organizational Bebavior and Human Decision Processes, 50, 179-211.

Arbuckle, J. L. (1996). Full information estimation in the presence of incomplete data. In G. A. Marcoulides \& R. E. Schumacker (Eds.), Advanced structural equation modeling (pp. 243-277). Mahwah, NJ: Erlbaum. 
Bandura, A. (2004). Health promotion by social cognitive means. Health Education and Bebavior, 31, 143-164.

Booth, M. L. (2000). Assessment of physical activity: An international perspective. Research Quarterly for Exercise and Sport, 71, 114-120.

Dusseldorp, E., Van Elderen, T., Maes, S., Meulman, J., \& Kraaij, V. (1999). A meta-analysis of psychoeducational programs for coronary heart disease patients. Health Psycbology, 18, 506-519.

Godin, G., \& Kok, G. (1996). The theory of planned behavior: A review of its applications to healthrelated behaviors. American Journal of Healtb Promotion, 11, 87-97.

Gollwitzer, P. M. (1999). Implementation intentions: Strong effects of simple plans. American Psychologist, 54, 493-503.

Gollwitzer, P. M., \& Sheeran, P. (2006). Implementation intentions and goal achievement: A metaanalysis of effects and processes. Advances in Experimental Social Psychology, 38, 69-119.

Hallam, J. S., \& Petosa, R. (2004). The long-term impact of a four-session worksite intervention on selected social cognitive theory variables linked to adult exercise adherence. Health Education and Behavior, 31, 88-100.

Hu, L., \& Bentler, P. M. (1998). Fit indices in covariance structure modeling: Sensitivity to underparameterized model misspecification. Psychological Methods, 3, 253-424.

Jackson, C., Lawton, R., Knapp, P., Raynor, D. K., Conner, M., Lowe, C., et al. (2005). Beyond intention: Do specific plans increase health behaviors in patients in primary care? A study of fruit and vegetable consumption. Social Science and Medicine, 60, 2383-2391.

Kline, R. B. (2005). Principles and practice of structural equation modelling. New York: Guilford Press.

Kraut, R., Olson, J., Banaji, M., Bruckman, A., Cohen, J., \& Couper, M. (2004). Psychological research online. Report of board of scientific affairs' advisory group on the conduct of research on the internet. American Psycbologist, 59, 105-117.

Lachman, M. E., \& Burack, O. R. (1993). Planning and control processes across the life span: An overview. International Journal of Bebavioral Development, 16, 131-143.

Larimer, M. E., Palmer, R. S., \& Marlatt, G. A. (1999). Relapse prevention: An overview of Marlatt's cognitive-behavioral model. Alcobol Research and Health, 23, 151-160.

Lee, I.-M., \& Paffenbarger, R. S. (1998). Physical activity and stroke incidence. The Harvard Alumni Health Study. Stroke, 29, 2049-2054.

Leventhal, H., Singer, R., \& Jones, S. (1965). Effects of fear and specificity of recommendation upon attitudes and behavior. Journal of Personality and Social Psychology, 2, 20-29.

Lippke, S., Ziegelmann, J. P., \& Schwarzer, R. (2004a). Behavioral intentions and action plans promote physical exercise: A longitudinal study with orthopedic rehabilitation patients. Journal of Sport and Exercise Psychology, 26, 470-483.

Lippke, S., Ziegelmann, J. P., \& Schwarzer, R. (2004b). Initiation and maintenance of physical exercise: Stage-specific effects of a planning intervention. Research in Sports Medicine, 12, 221-240.

Lippke, S., Ziegelmann, J. P., \& Schwarzer, R. (2005). Stage-specific adoption and maintenance of physical activity: Testing a three-stage model. Psychology of Sport and Exercise, 6, 585-603.

Luszczynska, A., \& Schwarzer, R. (2003). Planning and self-efficacy in the adoption and maintenance of breast self-examination: A longitudinal study on self-regulatory cognitions. Psychology and Health, 18, 93-108.

Maddux, J. E., \& Rogers, R. W. (1983). Protection motivation and self-efficacy: A revised theory of fear appeals and attitude change. Journal of Experimental Social Psycbology, 19, 469-479.

Marlatt, G. A. (1996). Taxonomy of high-risk situations for alcohol relapse: Evolution and development of a cognitive-behavioral model. Addiction, 91, 37-50.

Marlatt, G. A. \& Gordon, J. R. (Eds.). (1985). Relapse prevention: Maintenance strategies in the treatment of addictive bebaviors. New York: Guilford Press. 
Marsh, H. W., Wen, Z., \& Hau, K.-T. (2004). Structural equation models of latent interactions: Evaluation of alternative estimation strategies and indicator construction. Psychological Methods, 9, 275-300.

Miller, D. J., Freedson, P. S., \& Kline, G. M. (1994). Comparison of activity levels using the Caltrac accelerometer and five questionnaires. Medicine and Science in Sports and Exercise, 26, 376-382.

Milne, S., Orbell, S., \& Sheeran, P. (2002). Combining motivational and volitional interventions to promote exercise participation: Protection motivation theory and implementation intentions. British Journal of Health Psychology, 7, 163-184.

Norman, P., \& Conner, M. (2005). The theory of planned behavior and exercise: Evidence for the mediating and moderating roles of planning on intention-behavior relations. Journal of Sport and Exercise Psychology, 27, 488-504.

Orbell, S., \& Sheeran, P. (1998). 'Inclined abstainers' A problem for predicting health-related behavior. British Journal of Social Psychology, 37, 151-165.

Patterson, C. J., \& Mischel, W. (1976). Effects of temptation-inhibiting and task-facilitating plans on self-control. Journal of Personality and Social Psychology, 33, 209-217.

Prestwich, A., Conner, M., Lawton, R., Bailey, W., Litman, J., \& Molyneaux, V. (2005). Individual and collaborative implementation intentions and the promotion of breast self-examination. Psycbology and Healtb, 20, 743-760.

Rademacher, J. D. M., \& Lippke, S. (2007). Dynamic online surveys and experiments with the free open source software dynQuest. Behavior Research Methods, 39(3), 415-426.

Reips, U.-D. (2002). Standards for Internet-based experimenting. Experimental Psychology, 49 , 243-256.

Sallis, J. F., \& Saelens, B. E. (2000). Assessment of physical activity by self-reports: Status, limitations, and future directions. Research Quarterly for Exercise and Sport, 71, 1-14.

Schoenborn, C. A., Adams, P. F, Barnes, P. M., Vickerie, J. L., \& Schiller, J. S. (2004). Health behaviors of adults: United States, 1999-2001. National Center for Health Statistics. Vital and Healtb Statistics, 10(219), 1-79.

Scholz, U., Sniehotta, F. F, \& Schwarzer, R. (2005). Predicting physical exercise in cardiac rehabilitation: The role of phase-specific self-efficacy beliefs. Journal of Sport and Exercise Psychology, 27, 135-151.

Schwarzer, R. (1992). Self-efficacy in the adoption and maintenance of health behaviors: Theoretical approaches and a new model. In R. Schwarzer (Ed.), Selfefficacy: Thougbt control of action (pp. 217-243). Washington, DC: Hemisphere.

Schwarzer, R., Schüz, B., Ziegelmann, J. P., Lippke, S., Luszczynska, A., \& Scholz, U. (2007). Adoption and maintenance of four health behaviors: Theory-guided longitudinal studies on dental flossing, seat belt use, dietary behavior, and physical activity. Annals of Bebavioral Medicine, 33, 156-166.

Sesso, H. D., Paffenbarger, R. S., \& Lee, I.-M. (2000). Physical activity and coronary heart disease in men. The Harvard Alumni Health Study. Circulation, 102, 975-980.

Sheeran, P. (2002). Intention-behavior relations: A conceptual and empirical review. In M. Hewstone \&. W. Stroebe (Eds.), European review of social psychology (Vol. 12, pp. 1-36). Chichester, England: Wiley.

Sheeran, P., Milne, S., Webb, T. L., \& Gollwitzer, P. M. (2005). Implementation intentions and health behavior. In M. Conner \& P. Norman (Eds.), Predicting bealth behavior (2nd ed., pp. 276-323). Buckingham, England: Open University Press.

Sheeran, P., Webb, T. L., \& Gollwitzer, P. M. (2005). The interplay between goal intentions and implementation intentions. Personality and Social Psychology Bulletin, 31, 87-98.

Simkin, L. R., \& Gross, A. M. (1994). Assessment of coping with high-risk situations for exercise relapse among healthy women. Healtb Psycbology, 13, 274-277.

Sniehotta, F. F, Scholz, U., \& Schwarzer, R. (2005). Bridging the intention-behavior gap: Planning, self-efficacy, and action control in the adoption and maintenance of physical exercise. Psychology and Health, 20, 143-160. 
Sniehotta, F. F, Scholz, U., Schwarzer, R., Fuhrmann, B., Kiwus, U., \& Völler, H. (2005). Long-term effects of two psychological interventions on physical exercise and self-regulation following coronary rehabilitation. International Journal of Bebavioral Medicine, 12, 244-255.

Sniehotta, F. F., Schwarzer, R., Scholz, U., \& Schüz, B. (2005). Action planning and coping planning for long-term lifestyle change: Theory and assessment. European Journal of Social Psycbology, 35, 565-576.

Sobel, M. E. (1982). Asymptotic intervals for indirect effects in structural equations models. In S. Leinhart (Ed.), Sociological methodology 1982 (pp. 290-312). San Francisco, CA: JosseyBass.

Sutton, S. (2005). Stage theories of health behavior. In M. Conner \& P. Norman (Eds.), Predicting bealth behavior (2nd ed., pp. 223-275). Maidenhead, England: Open University Press.

U.S. Department of Health and Human Services (1996). Pbysical activity and bealtb: $A$ report of the surgeon general. Atlanta, GA: Centers for Disease Control and Prevention, National Center for Chronic Disease Prevention and Health Promotion.

Verplanken, B., \& Faes, S. (1999). Good intentions, bad habits and the effects of forming implementation intentions on behavior and cognition. European Journal of Social Psychology, 29, 591-604.

Weinstein, N. D., Rothman, A. J., \& Sutton, S. R. (1998). Stage theories of health behavior:

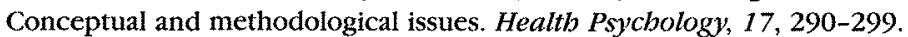

Ziegelmann, J. P., Lippke, S., \& Schwarzer, R. (2006). Adoption and maintenance of physical activity: Planning interventions in young, middle-aged, and older adults. Psychology and Health, 21, 145-163. 